\title{
Abdominal Pain as the Manifestation of Chylomicronemia Syndrome During Pregnancy: A Challenging Diagnosis
}

\author{
Catarina Parente ${ }^{1}$, Rúben Reis ${ }^{1}$, Manuel Toscano ${ }^{2}$, Isabel Botelho ${ }^{3}$, Armindo Ramos ${ }^{3}$ \\ 1. Internal Medicine, Centro Hospitalar Barreiro Montijo, Barreiro, PRT 2. Internal Medicine, Hospital De Cascais, \\ Cascais, PRT 3. Internal Medicine: Critical Care, Hospital De Cascais, Cascais, PRT
}

Corresponding author: Catarina Parente, catarina.parente6@gmail.com

\begin{abstract}
Hypertriglyceridemia is a frequent cause of acute pancreatitis. Levels higher than $1000 \mathrm{mg} / \mathrm{dL}$ are often associated with a genetic predisposition that can be aggravated by other factors such as pregnancy and poorly controlled diabetes.
\end{abstract}

The authors report a 19-year-old primigravida that presented with abdominal pain, emesis and a pruritic rash, along with severely increased plasma triglyceride levels. Therapeutic plasmapheresis was proposed in the setting of a presumed acute pancreatitis.

Chylomicronemia syndrome is a rare and frequently misdiagnosed pathology that can evolve with abdominal pain, vomiting and a specific cutaneous rash designated as eruptive xanthomatosis. This case report illustrates the challenges of achieving a correct diagnosis for rare conditions and corroborates the safety of plasmapheresis during pregnancy.

Review began 03/22/2021 Review ended 04/01/2021 Published 04/06/2021

๑) Copyright 2021 Parente et al. This is an open access article distributed under the terms of the Creative Commons Attribution License CC-BY 4.0., which permits unrestricted use, distribution, and reproduction in any medium, provided the original author and source are credited.
Categories: Endocrinology/Diabetes/Metabolism

Keywords: chylomicrons, triglycerides, atypical rash, acute pancreatitis, pregnancy, abdominal pain, plasmapheresis

\section{Introduction}

Abdominal pain is a common symptom frequently approached in the emergency room that can be attributed to multiple causes of distinct clinical severity. One potentially life-threatening condition that manifests with abdominal pain is acute pancreatitis, which is a well-known and frequent entity in clinical practice. Although its diagnosis can be relatively simple, determining the etiology might be challenging. The gold standard image exam for diagnosis of pancreatitis is contrast-enhanced computed tomography (CT), which also allows the exclusion of local complications. In the absence of complicated disease, the initial management usually consists of supportive care with fluid therapy, pain control and restriction of oral intake.

Another frequent cause of abdominal pain that typically occurs with nausea and vomiting is diabetic ketoacidosis, which is generally treated by supportive measures such as glycaemic control, intravenous hydration and correction of electrolytic and acid-base balance disturbances. In pregnancy, a whole other group of diagnostic hypotheses that have to be excluded emerges, including eclampsia and preeclampsia, HELLP (Hemolysis, Elevated Liver enzymes, Low Platelets) syndrome, and uterine contractions amongst others.

A rather rare cause of abdominal pain is chylomicronemia syndrome, which is manifested by a constellation of signs and symptoms: severe elevations in plasma triglycerides, abdominal pain, recurrent episodes of acute pancreatitis, eruptive xanthomatosis and lipemia retinalis [1]. The present case report represents the diagnostic complexity of abdominal pain in the setting of multiple confounding factors and the limitation of certain complementary exams due to gestation or unsuitable blood samples.

\section{Case Presentation}

A 19-year-old woman with a history of diabetes of unknown etiology, for which there was no therapeutic compliance or follow-up care, presented to the emergency department with nausea, emesis and diffuse abdominal pain. She had an estimated 27-week pregnancy that had not been monitored up to that point. There was no fever or other symptoms. A blood sample was drawn for laboratory analysis which was milky white in the syringe (Figure 1) and results were unobtainable. 


\section{Cureus}

\section{FIGURE 1: Syringe with blood sample}

Milky white appearance of collected blood sample (white arrow) from a peripheral vein

Arterial blood gas analysis revealed a pH of 7.12 and bicarbonate level of $3.3 \mathrm{mmol} / \mathrm{L}$, capillary blood glucose was $327 \mathrm{mg} / \mathrm{dL}$ and capillary ketone determination was $5 \mathrm{mmol} / \mathrm{L}$. The patient was admitted to the intermediate care unit for diabetic ketoacidosis and started treatment accordingly. Upon admission, a pruritic papular rash was noted in the patient's legs, feet, arms, hands and trunk.

After intravenous fluid therapy, it was possible to perform blood work which demonstrated a glycated haemoglobin level of 12\%, plasma amylase of $130 \mathrm{UI} / \mathrm{L}$ (reference range: 25 - $115 \mathrm{UI} / \mathrm{L}$ ) and triglycerides of $9755 \mathrm{mg} / \mathrm{dL}$. Moreover, the patient also had hypercholesterolemia with a total cholesterol of $1065 \mathrm{mg} / \mathrm{dL}$, high-density lipoprotein (HDL) < $20 \mathrm{mg} / \mathrm{dL}$ and low-density lipoprotein (LDL) of $374 \mathrm{mg} / \mathrm{dL}$ (direct

homogeneous enzymatic determination method). There were no detectable changes in abdominal and fetal ultrasound and no contractility in cardiotocography. The case was interpreted as acute pancreatitis due to severe hypertriglyceridemia, and plasmapheresis was conducted (Figure 2). After one session of approximately four hours, her serum triglycerides level was $559 \mathrm{mg} / \mathrm{dL}$ and her symptoms resolved. She then started treatment with gemfibrozil. 


\section{Cureus}

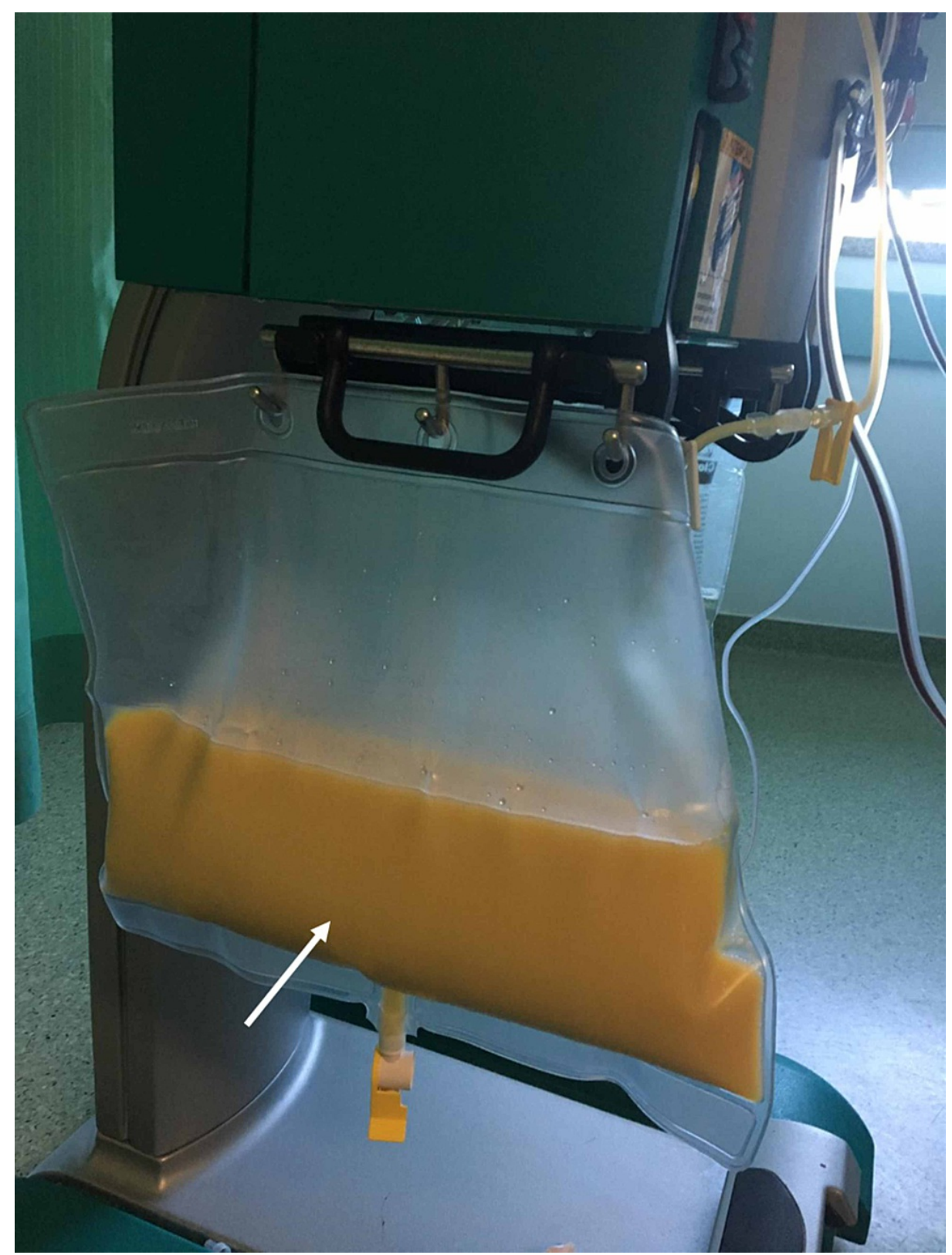

\section{FIGURE 2: Plasmapheresis system}

Milky white appearance of plasma (white arrow) in the processing bag

A hypercholesterolemia study was carried out with apolipoprotein A1 assay value within the reference range and with apolipoproteins E and C-II slightly elevated, $73.6 \mathrm{mg} / \mathrm{dL}$ (reference range: 23.0 - 63.0) and 9.5 $\mathrm{mg} / \mathrm{dL}$ (reference range: 1.6 - 4.2), respectively. It was not possible to measure apolipoprotein B because the blood sample was too lipemic.

The patient was discharged to pursue medical care and investigation in ambulatory regimen but was not compliant to follow-up and further investigation was not possible.

\section{Discussion}

Chylomicronemia syndrome (CS) is a largely misdiagnosed condition characterized by severe elevations in plasma triglycerides, often greater than $1000 \mathrm{mg} / \mathrm{dL}$, and by clinical features as recurrent episodes of abdominal pain that may be associated with nausea and vomiting, most common presenting symptom, acute pancreatitis, lipemia retinalis [1] and/or hepatosplenomegaly. A skin rash may be present in the form of various dispersed papular lesions, yellow, pink or brown coloured, designated as eruptive xanthomatosis which can be pruritic or occasionally painful [2]. 
There are two forms of CS: familiar chylomicronemia syndrome (FCS), related to mutations affecting the enzyme lipoprotein lipase or its regulators, apolipoprotein C-II, apolipoprotein A5, GPIHBP1 (Glycosylphosphatidylinositol Anchored High Density Lipoprotein Binding Protein 1) and LMF1 (Lipase Maturation Factor 1); and multifactorial chylomicronemia syndrome (MCS) which results from minor polygenic variants and is exacerbated by other medical conditions [3].

Genetic testing for FCS is limited since it is only available at specialized laboratories and it may wrongly dismiss some patients because not all mutations are known [4]. Nevertheless, its diagnosis can be established predominately in the clinical setting $[4,5]$ reuniting the following criteria: severe hypertriglyceridemia (> $880 \mathrm{mg} / \mathrm{dL}$ ) in three determinations refractory to treatment and diet; history of acute pancreatitis or recurrent abdominal pain, absence of familial history of combined hyperlipidaemia, age of onset of symptoms and exclusion of secondary causes (except pregnancy and ethinylestradiol treatment) [5].

The patient presented with diffuse abdominal pain, nausea and vomiting in the setting of uncontrolled diabetes, namely manifested as diabetic ketoacidosis, and triglycerides level > $9000 \mathrm{mg} / \mathrm{dL}$, associated with a pruritic papular skin rash. Even though eruptive xanthomatosis could not be confirmed due to the absence of a skin biopsy, CS prevailed as the most suitable diagnosis reuniting the presentation features that were, as previously mentioned, severely increased plasma triglycerides, abdominal pain, nausea and vomiting, although some of these symptoms could be attributed to diabetic ketoacidosis. Regardless, acute pancreatitis cannot be excluded since it can occur in the context of CS and considering amylase levels may be falsely normal in the context of hypertriglyceridemia [6]. On the other hand, nonspecific elevations of amylase and lipase can occur in diabetic ketoacidosis [7]. It should be noted that a CT scan was not performed to further investigate this hypothesis due to the potential risks to the foetus. Moreover, all these conditions are not mutually exclusive and can occur simultaneously comprising a rare triad that has been previously described in medical literature.

Despite the well-defined chylomicronemia syndrome (with or without acute pancreatitis), the distinction between FCS and MCS may be more complex. The young age, low body weight and extremely elevated triglyceride levels with a milky white plasma appearance which is a key characteristic of FCS [5] favor this hypothesis. Conflictingly, the patient had uncontrolled diabetes as a precipitating factor and her past medical history was unremarkable for acute pancreatitis. Furthermore, there were no prior triglyceride level determinations.

Considering genetic testing was not immediately available and there was not enough data for using the mentioned clinical criteria, a definitive cause of CS, familiar or multifactorial, could not be concluded.

Another aspect of this case report is the use of plasmapheresis. Therapeutic plasma exchange is a safe and effective technique that can be performed in a broad spectrum of diseases, including during pregnancy, and it is recommended as a second-line therapy in hypertriglyceridemia-induced acute pancreatitis in pregnant women. An important consideration while implementing this procedure in pregnant patients is to ensure a correct estimation of blood and plasma volume (which will be increased especially in the second and third trimesters) [8].

\section{Conclusions}

The reported clinical case was challenging considering that abdominal pain, nausea and vomiting can occur in diabetic ketoacidosis, as well as in acute pancreatitis and in chylomicronemia syndrome manifestation. Amylase levels were borderline normal and a lipase level was not obtained prior to plasmapheresis. Concomitantly, a CT scan was not performed to clarify the diagnosis due to the ongoing pregnancy. Nevertheless, all three conditions could be present simultaneously consisting of a rare but previously described triad in literature.

We highlight the diagnostic difficulty of abdominal pain in the presence of confounding factors in the routine clinical practice, as well as the use of plasmapheresis for the treatment of severe hypertriglyceridemia in pregnancy, which was performed without reportable complications.

Furthermore, the authors emphasize that a patient's lack of compliance to treatment and follow-up can be truly limiting for medical action and clinicians should be aware of this problem so that education and sensitization strategies can be developed.

\section{Additional Information \\ Disclosures}

Human subjects: Consent was obtained or waived by all participants in this study. Conflicts of interest: In compliance with the ICMJE uniform disclosure form, all authors declare the following: Payment/services info: All authors have declared that no financial support was received from any organization for the submitted work. Financial relationships: All authors have declared that they have no financial relationships at present or within the previous three years with any organizations that might have an 


\section{Cureus}

interest in the submitted work. Other relationships: All authors have declared that there are no other relationships or activities that could appear to have influenced the submitted work.

\section{References}

1. Warden BA, Minnier J, Duell PB, Fazio S, Shapiro MD: Chylomicronemia syndrome: Familial or not? J Clin Lipidol. 2020, 14:201-206. 10.1016/j.jacl.2020.01.014

2. Kashif M, Kumar H, Khaja M: An unusual presentation of eruptive xanthoma: A case report and literature review. Medicine (Baltimore). 2016, 95:e4866. 10.1097/MD.0000000000004866

3. Goldberg RB, Chait A: A Comprehensive Update on the Chylomicronemia Syndrome . Front Endocrinol (Lausanne). 2020, 11:593931. 10.3389/fendo.2020.593931

4. Falko JM: Familial Chylomicronemia Syndrome: A Clinical Guide For Endocrinologists . Endocr Pract. 2018, 24:756-763. 10.4158/EP-2018-0157

5. Moulin P, Dufour R, Averna M, et al.: Identification and diagnosis of patients with familial chylomicronaemia syndrome (FCS): Expert panel recommendations and proposal of an "FCS score". Atherosclerosis. 2018, 275:265-272. 10.1016/j.atherosclerosis.2018.06.814

6. Melnick S, Nazir S, Gish D, Aryal MR: Hypertriglyceridemic pancreatitis associated with confounding laboratory abnormalities. J Community Hosp Intern Med Perspect. 2016, 6:31808. 10.3402/jchimp.v6.31808

7. Yadav D, Nair S, Norkus EP, Pitchumoni CS: Nonspecific hyperamylasemia and hyperlipasemia in diabetic ketoacidosis: incidence and correlation with biochemical abnormalities. Am J Gastroenterol. 2000, 95:31238. 10.1016/S0002-9270(00)02072-4

8. Wind M, Gaasbeek AGA, Oosten LEM, Rabelink TJ, van Lith JMM, Sueters M, Teng YKO: Therapeutic plasma exchange in pregnancy: A literature review. Eur J Obstet Gynecol Reprod Biol. 2021, 260:29-36.

10.1016/j.ejogrb.2021.02.027 